Port Acadie

Revue interdisciplinaire en études acadiennes

An Interdisciplinary Review in Acadian Studies



\title{
Les prêtres et religieux auteurs de chansonniers
}

\section{Marcel Bénéteau}

Numéro 24-25-26, automne 2013, printemps-automne 2014

L'Apport des prêtres et des religieux au patrimoine des minorités : parcours comparés Bretagne/Canada français

URI : https://id.erudit.org/iderudit/1019137ar

DOI : https://doi.org/10.7202/1019137ar

Aller au sommaire du numéro

Éditeur(s)

Université Sainte-Anne

ISSN

1498-7651 (imprimé)

1916-7334 (numérique)

Découvrir la revue

Citer cet article

Bénéteau, M. (2013). Les prêtres et religieux auteurs de chansonniers. Port Acadie, (24-25-26), 258-272. https://doi.org/10.7202/1019137ar

\section{Résumé de l'article}

Les prêtres et religieux s'intéressent de longue date à la chanson populaire, autant comme " ponton de sauvetage " et « bain d'énergie patriotique " que comme forme d'expression à perfectionner, à réhabiliter et à consacrer. Quel est l'apport du clergé au répertoire canadien ? L'ensemble de leurs efforts a-t-il diminué ou augmenté le rayonnement de la chanson traditionnelle dans la culture populaire ? Y a-t-il une continuité entre les premiers chansonniers d'écoles et de collèges, souvent compilés par des membres du clergé, les " améliorations » des abbés Burque et Gadbois et les sélections se voulant plus scientifiques publiées par Anselme Chiasson et Germain Lemieux? 


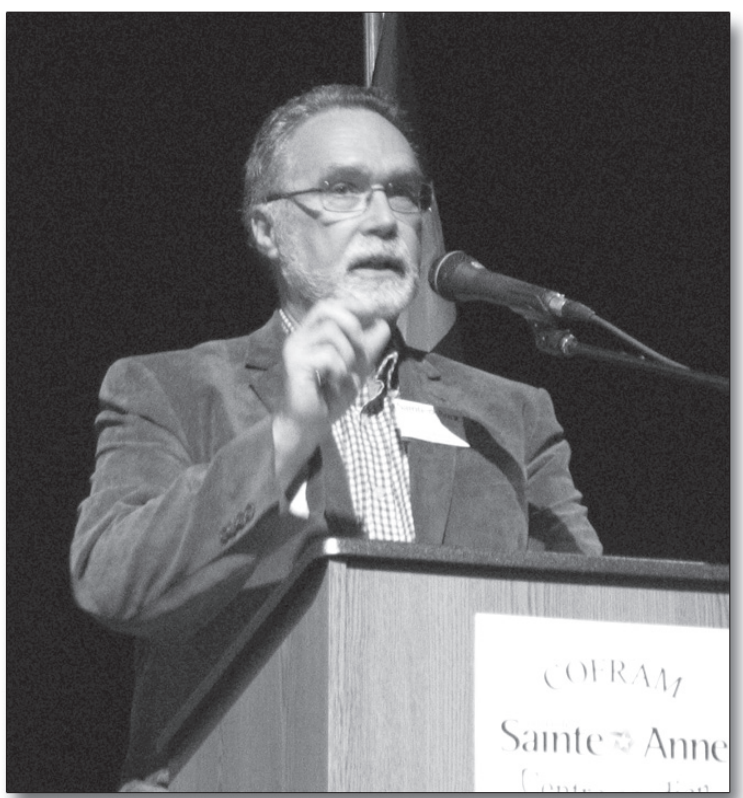

Marcel Bénéteau 


\title{
Les prêtres et religieux auteurs de chansonniers
}

\author{
Marcel Bénéteau \\ Université de Sudbury
}

\begin{abstract}
Résumé
Les prêtres et religieux s'intéressent de longue date à la chanson populaire, autant comme " ponton de sauvetage » et " bain d'énergie patriotique » que comme forme d'expression à perfectionner, à réhabiliter et à consacrer. Quel est l'apport du clergé au répertoire canadien ? L'ensemble de leurs efforts a-t-il diminué ou augmenté le rayonnement de la chanson traditionnelle dans la culture populaire ? Y a-t-il une continuité entre les premiers chansonniers d'écoles et de collèges, souvent compilés par des membres du clergé, les " améliorations » des abbés Burque et Gadbois et les sélections se voulant plus scientifiques publiées par Anselme Chiasson et Germain Lemieux?
\end{abstract}

Le phénomène que je veux examiner ici s'éloigne un peu de la notion de "prêtres collecteurs ", puisque je vais me pencher sur des personnes qui ont été plutôt des " compilateurs » ou des "rassembleurs " que de véritables collecteurs. En choisissant de publier des " chansonniers ", certains prêtres et religieux ont produit des documents qui, de par leur définition même, ont comme but premier de faire chanter ; et comme nous le verrons, ce que l'on chante, selon ces auteurs, n'est pas sans conséquences. Le "choix de chansons », pour reprendre le titre du recueil du frère Charles-Borromée Perreault ${ }^{1}$, peut servir à des fins idéologiques et n'a rien de l'esprit de la recherche scientifique ou même la curiosité intellectuelle qui a motivé plusieurs prêtres collecteurs. Toutefois, il ne faut pas être trop catégorique en opposant les buts et motivations des compilateurs de chansonniers à celles des chercheurs : il est clair que les deux groupes ont œuvré au moins en partie dans l'intention de sauver un patrimoine qu'ils considéraient menacé et en voie de disparition. Mais il y a tout un monde de différence entre la description et la mise en valeur de ce qui existe et la consécration et la canonisation de ce qui devrait être.

Pourtant les deux approches ont eu un effet sur la sauvegarde et la mise en valeur d'un répertoire folklorique et sur la notion même de ce qui est «folklore » et « culture populaire ». Les compilateurs de chansonniers ont eu une influence certaine chez les folkloristes et, au Canada français, les ont même précédés : par exemple, selon Conrad Laforte², le

1.

Charles-Borromée Perreault, Choix de chansons, Montréal, Pensionnat MontSaint-Louis, 1914, 219 p.

2. Conrad Laforte, La Chanson folklorique et les écrivains du xix siècle (en France et au Québec), Montréal, Éditions Hurtubise HMH, «Ethnologie québécoise, Les Cahiers du Québec », 1973, 154 p. 
Chansonnier des collèges ${ }^{3}$, que nous examinerons sous peu, aurait été la source de plusieurs chants publiés par Hubert La Rue dans « Les Chansons populaires et historiques du Canada 4 », considérés à juste titre la première étude de chansons folkloriques au Canada français. Laforte affirme aussi que le compilateur du Chansonnier, l'abbé Charles-Honoré Laverdière, aurait lui-même été un des informateurs d'Ernest Gagnon, donnant quatre ou cinq chansons aux Chansons populaires du Canada ${ }^{5}$. Les chansonniers ont d'ailleurs connu une bien plus grande diffusion que les travaux des folkloristes ; par exemple, les cinq éditions du Chansonnier des collèges, chacune plus volumineuse que la précédente, ont été pillées par tous les chansonniers scolaires subséquents, y compris le Chansonnier des écoles, ouvrage autorisé par la Commission des écoles catholiques romaines de Montréal en $1876^{6}$. Le Choix de chansons de Borromée Perreault a connu six éditions de 1909 à 1942. Et rien ne surpasse, au Canada français, l'empire de la Bonne Chanson de l'abbé Gadbois7. On peut donc se demander : quelle a été l'influence des chansonniers sur le répertoire canadien ? A-t-elle diminué ou augmenté le rayonnement de la chanson traditionnelle dans la culture populaire?

Nous ne pourrons examiner dans le cadre de cette communication tous les chansonniers publiés par des prêtres et religieux ; je fais donc mon propre « choix de chansonniers ». Je vais miser d'abord sur un des premiers, déjà mentionné : Le Chansonnier des collèges de l'abbé Laverdière, publié d'abord en 1850. Ensuite je traiterai brièvement d'un de ses émules, le frère Charles-Borromée Perreault, qui publie son Choix de chansons en 1909. Je m'attarderai par la suite sur deux compilateurs aussi influents que controversés du Canada français au xxe siècle : l'abbé F.-X. Burque et l'abbé Charles Gadbois. En conclusion, je ferai quelques observations sur le travail d'un prêtre collecteur et folkloriste qui a aussi publié un « choix de

3. Charles-Honoré Laverdière, Le Chansonnier des collèges, Québec, Au bureau de l'Abeille, 1850, 200 p. ; deuxième édition, révisée et augmentée, 1854, 328 p. + Supplément, $104 \mathrm{p}$.

4. $\quad$ François-Alexandre-Hubert La Rue, "Les Chansons populaires et historiques du Canada », dans Le Foyer canadien, Québec, vol. 1, 1863, p. 321-384; et "Les Chansons historiques du Canada », ibid., vol. 3, 1865, p. 5-72.

5. Ernest Gagnon, Chansons populaires du Canada recueillies et publiées avec annotations, etc., Québec, Bureau du Foyer canadien, 1865-1867.

6. Pour une analyse des divers manuels de chansons utilisés dans les écoles, voir Jean-Nicolas De Surmont, « Panorama du corpus chansonnier en contexte scolaire et de l'éducation à la jeunesse au Québec : quelques jalons pédagogiques et idéologiques ", dans Jeunesse : Young People, Texts, Cultures, vol. 2, $\mathrm{n}^{\circ} 1$, p. 71-83.

7. Connu, entre autres, à travers sa série des cahiers de La Bonne Chanson, SaintHyacinthe, Éditions de la Bonne Chanson, 1938-1954, 10 vol. 
chansons » dans des chansonniers destinés au grand public, c'est-à-dire le père Germain Lemieux.

\section{Le Chansonnier des collèges, un premier modèle}

Le Chansonnier des collèges, recueil compilé et édité par Charles-Honoré Laverdière, est un des premiers chansonniers rassemblés par un religieux au Canada français et il est demeuré un des plus influents. Laverdière est né en 1826 à Château-Richer et entré au petit séminaire de Québec en $1840^{8}$. Il devient assistant professeur au séminaire en 1948 et professeur de physique en 1850. Il est ordonné prêtre l'année suivante ; il publie donc la première édition du Chansonnier des collèges avant de prononcer ses derniers vœux. Connu surtout comme historien, il occupe la chaire d'histoire à l'Université Laval à partir de 1867 et participe à l'édition des Relations des jésuites et aussi des Fuvres de Champlain. Il est aussi bibliothécaire de l'Université. Quand il était étudiant, il avait participé à la fondation d'un journal d'étudiant, L'Abeille, dans lequel il continua à publier jusqu'à sa mort en 1873. C'est effectivement « au bureau de l'Abeille » qu'il publie son Chansonnier. On retire très peu d'information sur ses activités musicales dans les sources biographiques; le Dictionnaire biographique $d u$ Canada se contente de mentionner que, en plus de des travaux historiques, il publie " plusieurs recueils de cantiques profanes et religieux. »

La première édition du Chansonnier des collèges comprend les textes de 138 chansons, dont seulement cinq ou six peuvent être considérées comme venant de la tradition orale - entre autres Cadet Rousselle, La Palisse et Malbrough s'en va-t-en guerre. Les versions, en particulier de La Palisse (attribuée à Bernard de la Monnoye) et de Malbrough (" Mort et convoi de l'invincible Malbrough ») ont une forte saveur littéraire. Le reste du recueil consiste en des compositions modernes sinon contemporaines, canadiennes aussi bien que françaises, qui se regroupent autour de certains thèmes bien circonscrits : patriotisme, religion, nostalgie et sentiment : on y retrouve des odes au clocher du village, au hameau paisible, au laboureur laborieux, au vieux soldat, aux orphelins et mères mourantes. L'apport de la tradition orale est franchement négligeable, mais, dès la deuxième édition de 1854, un supplément de 88 pages apporte cette fois-ci 44 chansons de tradition orale. Les sources de ces chansons sont inconnues, mais on peut supposer que, comme d'autres compilateurs de l'époque, Laverdière se fie à sa propre mémoire, à celle de son entourage et à des recueils déjà publiés. Le supplément n'est pas sans intérêt pour les folkloristes, mettant en vedette trois versions de chacune de ces chan-

8. Les informations biographiques qui suivent sont tirées du Dictionnaire biographique du Canada en ligne, vol. x, 1871-1880, http://biographi.ca/oogoo4119.01-f.php?id_nbr=5091. 
sons : Trois Beaux Canards, Trois Cavaliers fort bien montés et Mon père a fait bâtir maison. On reste quand même parmi les chansons les mieux connues : Ah! si mon moine, Bonhomme sais-tu jouer, Il était une bergère. La boisson, le clergé et les nuits de noces sont notamment absents du répertoire. Une version du Meunier et la belle ne trahit aucune trace de grivoiserie. Évidemment, on n'y saisit qu'une partie minime du répertoire traditionnel chanté par les Canadiens.

Les chants traditionnels sont d'ailleurs présentés dans un ensemble de chansons « correctes " proposant un divertissement sain et propre pour tout étudiant. Le but ici n'est pas la sauvegarde d'un patrimoine, mais pour citer Jean-Nicolas De Surmont - de « répandre une vision de la morale et éduquer la jeunesse par la chanson », dans le cadre d'une littérature « didactique, moralisatrice, nationale et romantique ${ }^{9}$ ». Laverdière explique ainsi le besoin d'un tel recueil dans la préface de la première édition :

Il est vrai que, outre les chansonniers étrangers, il existe plusieurs recueils imprimés au Canada ; mais ordinairement, et pour cause, les chansonniers sont saisis à la douane du collège... C'est pour remédier à de si graves inconvénients que nous commençons aujourd'hui la publication du Chansonnier des collèges où nous tâcherons de réunir toutes les chansons les plus propres à charmer nos loisirs. ${ }^{10}$

\section{Le Choix de chansons}

Une génération plus tard, la visée des chansonniers n’a guère changé, comme on peut le constater par un aperçu, à titre d'exemple, du petit bouquin publié par le frère Charles Borromée Perreault. Né à Sainte-Monique, Nicolet, en 1880, Perreault entre chez les frères des Écoles chrétiennes en 1896. Il sera professeur au Mont Saint-Louis jusqu'à sa mort en $1924^{11}$. Il publie un livre de cantiques et aussi un recueil de "chansons profanes ", Choix de chansons en 1909 ; ce recueil sera réimprimé en 1914, en 1916, en 1924, en 1936 et en 1942. S'il y a innovation ici, c'est dans l'ajout d'un bon nombre de textes et musiques de Théodore Botrel, créateur du mouvement de la Bonne Chanson en France. Perreault avait reçu l'autorisation de Botrel de " puiser dans ses œuvres »; il ne faut donc pas s'étonner d'y voir foisonner les mêmes thèmes de la foi, de la langue et de la patrie, le tout baignant dans une glorification de la vie paysanne et à forte saveur

9. De Surmont, op. cit., « Panorama du corpus chansonnier... », p. 74.

10. Laverdière, op. cit., Préface, p. 2.

11. La seule source d'information biographique pour Perreault se trouve dans Un demi-siècle au Mont-Saint-Louis - 1888-1938 - L'album jubilaire, Montréal, Imprimerie De-La-Salle, 1939 (647 p.), p. 201. 
antimoderniste. La page de titre présente en guise de dédicace un texte du maître lui-même, ostensiblement composé pour son public canadien ; exhortant "l'ardente jeunesse » à chanter, aimer, croire et travailler. Les paroles de Botrel résument bien le contenu du recueil :

Chante, libre sous les grands cieux,

La Foi, l'Amour et la Patrie ;

Mêle les chants de Crémazie

Aux refrains naïfs des aïeux !

Le frère Perreault suit la recette proposée : parmi les 142 chansons françaises et les 6 anglaises qu'on y retrouve (dont God Save the King et The Maple Leaf), il inclut des textes de Crémazie, ainsi que de Louis Fréchette et Pamphile Lemay, parmi les chansons de Botrel et autres compositeurs contemporains. Bien que le bouquin ne soit accompagné d'aucun commentaire de son compilateur, son but est clairement l'édification de la jeunesse. Perreault y mélange des thèmes carrément de la mère patrie - Jeanne d'Arc au bûcher, Les Montagnards - à d'autres qui sont exclusivement canadiens - La Cabane à sucre, Vive la raquette! Parmi les "refrains naïfs des aïeux », il inclut une vingtaine de chansons de tradition orale, dont plusieurs avaient déjà paru dans le recueil de Laverdière (Trois Beaux Canards, Malbrough s'en va-t-en guerre, Mon père a fait bâtir maison, À la claire fontaine, entre autres), tout à fait aussi inoffensives que les versions de son prédécesseur. Ce qu'on peut cependant noter avec intérêt est un renforcement de la tendance à relier « folklore » à tout ce qui est ancien, rural, nostalgique, religieux et patriotique, courant qui va atteindre son apogée dans l'œuvre des deux principaux compilateurs du vingtième siècle : François-Xavier Burque et Charles-Émile Gadbois.

\section{Le Nouveau Chansonnier canadien-français}

En 1921 paraît le recueil de l'abbé François-Xavier Burque, le Nouveau Chansonnier canadien-français. Burque est né en 1851 à Saint-Hyacinthe, au Québec. Les détails biographiques sont rares sur ce personnage marquant dans le domaine de la chanson populaire au Canada français. Franc admirateur de la chanson de tradition populaire avec ses mots simples et expressifs et ses mélodies s'inspirant du chant du rossignol - qu'il hausse au rang de " patrimoine sacré »- il est déterminé à lui accorder une plus grande place dans le répertoire national qu'il considère en danger de pollution par la culture populaire contemporaine. II estime que cette contamination a cours surtout dans les villes et observe que les bonnes vieilles chansons subsistent surtout à la campagne. Mais c'est ici que son analyse de la situation et des solutions à prendre devient plus ou moins 
incohérente : selon lui, les chansons traditionnelles sont menacées en ville à cause de leur trop grande "rusticité »-donc les qualités mêmes qui les rendent dignes d'être conservées sont la cause du dédain que les classes supérieures éprouvent en leur présence. Il donne comme exemple Vive la Canadienne, dont la version populaire contient, selon lui, un seul bon couplet, le reste comportant des « ineffabilités de débauche et d'ivrognerie ${ }^{12}$ » devant lesquelles il ne faut pas s'étonner du fait que les gens cultivés l'aient chassée de leur répertoire. II s'attaque directement aux folkloristes, mettant même le travail d'Ernest Gagnon en cause : « La trop grande rusticité de quelques-unes des chansons de Monsieur Ernest Gagnon [...] ont fait un tort très réel et très grave à notre patrimoine de vieilles chansons dans son ensemble. ${ }^{13}$ » On retrouve effectivement dans le chansonnier 14 chansons de Gagnon " corrigées, refaites ou complétées par le compilateur ${ }^{14}$ ». Il ajoute que la tradition orale en général contient « une foule de vieilles chansons défectueuses à tous les degrés comme à tous les points de vue, et méritant cependant l'honneur d'être ainsi restaurées et conservées..$^{15}$ " L'abbé Burque inclut une cinquantaine de chansons traditionnelles parmi son recueil de chants patriotiques, religieux et nostalgiques, qu'il classe à trois niveaux : première classe - chansons à peine retouchées (entre autres, À la claire fontaine, Malbrough, Marie-Anne s'en va-t-au moulin). La deuxième classe comprend des chansons plus ou moins profondément modifiées et dont le texte primitif, reconnaissable encore, se confond entièrement ou presque entièrement avec le texte nouveau (La Guignolée, Dans les chantiers nous hivernons, La Fille aux oranges, etc.). Et la troisième classe - chansons tout à fait originales ou dont le texte primitif a été tellement refondu qu'on peut dire qu'il a complètement disparu dans le texte nouveau ; cette catégorie comprend surtout des chansons littéraires. Cette procédure, affirme-t-il, assurera que « toutes les chansons contenues dans notre Recueil peuvent se chanter partout, même dans les salons, au milieu des classes cultivées, sans donner prise au dégoût, à la répugnance qu'inspire généralement [...] une trop grande rusticité d'expression, ou une trop grande défectuosité de rime et de mesure, dans une foule de nos vieilles chansons démodées. ${ }^{16}$ " II faudrait donc, paraît-il, détruire la chanson traditionnelle afin de la sauver.

12. Burque, op. cit., p. Iv-v ; voir l'Annexe । pour une comparaison des textes de Gagnon et de Burque.

13. Ibid., p. Ix ; voir l'Annexe ॥ pour une comparaison de la version de Par derrière chez ma tante de Gagnon et la version « corrigée, refaite et complétée » de l'abbé Burque.

14. Loc. cit.

15. Loc. cit.

16. Burque, ibid., p. $x$. 
Il ne faut pas oublier que Burque publie son chansonnier à l'époque même où Marius Barbeau est engagé dans ses travaux sur la chanson traditionnelle et qu'un échantillonnage de sa collecte a déjà paru dans le Journal of American Folklore. Mais Burque, qui est parfaitement au courant des tendances de l'époque, déclare en fait la guerre aux folkloristes. Pour lui, les variations introduites par les chanteurs individuels constituent non une richesse, mais le défaut principal du folklore. Je me permets de citer un long passage qui conclut son argument :

[...] d'où vient que tant de chansons populaires sont si pauvres, littérairement parlant ? [...] cela est dû à une double cause : la première, c'est que le texte primitif, à force de passer de bouche en bouche et de main en main, a fini par dégénérer ; la seconde, c'est que beaucoup de compositeurs de chansons ne connaissent pas mieux, ou s'ils connaissent mieux, ne se donnent pas la peine de faire mieux [...] Faut-il que le monde littéraire soit l'esclave de ces deux piètres causes d'imperfections ? Nous ne le pensons pas. Ce serait pousser trop loin le culte du folklore.

Ce serait du caprice, pour ne pas dire un goût dépravé.

Tant pis pour toutes ces ébauches imparfaites écloses comme des champignons. Si elles n'en valent pas la peine, on ne les corrigera point ; éphémère sera leur vie. Si elles en valent la peine, le meilleur service à leur rendre, c'est de leur passer dessus la brosse ou le balai, pour les débarrasser des chenilles et vilains vers qui les tuent. ${ }^{17}$

C'est presque trop facile pour des gens de sensibilité moderne et de goût dépravé de balayer à leur tour les arguments d'un François-Xavier Burque. Mais son chansonnier a eu une diffusion large et une influence importante, non seulement sur le grand public, mais aussi chez celui qui se montrerait bientôt le plus fidèle adepte de la chanson populaire sous une main institutionnelle : l'abbé Charles-Émile Gadbois, directeur de l'entreprise de la Bonne Chanson au Canada.

\section{Les cahiers de La Bonne Chanson}

Je ne reprendrai pas ici tous les propos analysés dans l'étude de Jean-Nicolas De Surmont sur La Bonne Chanson, avec le sous-titre évocateur Le commerce de la tradition en France et au Québec dans la première

17.

Burque, ibid., p. xvI-xvII. 
moitié du xxe siècle ${ }^{18}$. De Surmont montre comment Gadbois s'inspire du travail de Botrel et se détermine à continuer son œuvre au Canada français ; il est également inspiré par l'approche de l'abbé Burque, rendant un hommage particulier à son traitement de Vive la Canadienne ${ }^{19}$. Gadbois, né à Saint-Hyacinthe en 1906, est ordonné prêtre en 1937. Il aura un plus grand succès que tous ces prédécesseurs; en plus de ses intérêts musicaux et ses penchants idéologiques, il a décidemment la bosse des affaires, exploitant avec assurance les nouvelles technologies de la radio et du disque et faisant appel à un vaste réseau de connaissances pour promouvoir son produit. Mais ce sont ses 10 cahiers de La Bonne Chanson, publiés de 1938 à 1954, qui demeurent au cœur de l'entreprise : déjà en 1955, la série avait connu une très grande diffusion aux quatre coins du monde ${ }^{20}$. Gadbois vise les foyers canadiens, les écoles, les sociétés religieuses et missionnaires; il a l'appui du clergé, de l'élite politique, du Congrès de la langue française au Québec et du Comité catholique du Conseil de l'instruction publique. II crée donc un mariage parfait de marketing et d'idéologie.

Son succès est tel que La Bonne Chanson sera désormais indissociablement liée au folklore dans l'imaginaire populaire, désignée par la société des entreprises culturelles du gouvernement du Québec comme « le répertoire le plus complet de chants folkloriques canadiens-français ${ }^{21}$ ». En fait, les cahiers de La Bonne Chanson contiennent très peu de folklore - à peine $10 \%$, du total, si l'on fait le calcul. Et ce $10 \%$ est pour la plupart censuré

18. Jean-Nicolas De Surmont, La Bonne Chanson. Le commerce de la tradition en France et au Québec dans la première moitié du xxe siècle, Montréal, Les éditions Triptyque, 2001, $215 \mathrm{p}$.

19. En reprenant la version de Burque, Gadbois reprend également la citation de Benjamin Sulte, parue dans le Monde Illustré de Montréal, le 22 octobre 1892 : "L'idée de M. l'abbé Burque, de développer le chant de "Vive la Canadienne" a été excellente et tout à fait dans l'ordre. Il était temps qu'un Canadien à l'esprit élevé comme l'est $M$. Burque se déterminât à concéder plus de terrain aux vertus de ce sexe auquel nous devons nos mères et nos sœurs. Dorénavant, on chantera la "Canadienne" plus longtemps et ce sera pour le mieux. » (La Bonne Chanson, vol. 2, p. 76). À noter que Gadbois publie aussi dans le même numéro la composition de Burque, "Vive le Canadien ", personnage duquel on peut dire, au refrain d'En roulant ma boule, qu'il est «Bon citoyen, fort bon vivant/II s'enrichit en cultivant » et que «Tout plein de foi, le cœur fervent/À son église il va souvent. »

20. De Surmont, op. cit., p. 75-76: l'auteur avance le nombre fabuleux de cent trente millions d'exemplaires de chansons...

21. Communiqué des Entreprises culturelles enr., 4 juin 1979 ; cité par De Surmont, ibid., p. 77. Voir aussi De Surmont, « Les conditions de production et de circulation de l'œuvre de La Bonne Chanson de Charles-Émile Gadbois », dans Les Cahiers de l'association pour l'avancement de la recherche en musique au Québec, $\mathrm{n}^{\circ} 16$, p. 65-78, pour un compte rendu de la création et de la croissance de l'entreprise de La Bonne Chanson. 
et édulcoré, suivant l'exemple de l'abbé Burque. Ce qui est choisi s'insère bien dans la thématique générale de la primauté de la religion, l'autorité du père, l'importance du foyer, l'idéalisation des héros et un rejet complet de la modernité. Selon Gadbois, ses cahiers de chansons combattent « les chants qui se font remarquer par leur pauvreté ou leur mauvais goût musical, ou par la grivoiserie et l'immoralité de leurs propos ${ }^{22}$ ». (Notons que même les chansons de Félix Leclerc sont qualifiées d'une «insignifiance sans nom » par le bon abbé.) Gadbois cherche à établir un répertoire sain et moral qui sera chanté dans les foyers, dans les écoles, par un peuple qui se rattache aux anciennes valeurs de leurs aïeux. Il reprend souvent une citation de Paul Claudel pour justifier ses efforts : " Le jour où la musique mécanique ou le dur jazz américain vous aura fait oublier la parole vivante de vos pères, ce jour sera un triste jour pour la Nouvelle-France et l'Acadie et j'espère de tout mon cœur qu'il ne viendra jamais. "

Somme toute, on peut conclure que les chansonniers publiés par les prêtres et religieux des $x x^{e}$ et $x x^{e}$ siècles ont sans doute mené à établir un certain canon de chansons folkloriques et à assurer leur survie et leur grande popularité dans le répertoire chanté par la population canadiennefrançaise ; encore aujourd'hui, les chansons recueillies les plus souvent par nos étudiants témoignent de cette intronisation de certains titres du répertoire - Trois Beaux Canards, À la claire fontaine, Ah ! si mon moine voulait danser, À Saint-Malo, etc. ${ }^{23}$ Mais en éliminant une vaste quantité de chansons jugées « inappropriées », en réduisant le nombre de versions " imparfaites » et en les remplaçant par des versions fades et épurées, il ne semble pas exagéré de suggérer que le résultat net aurait vidé le folklore de son caractère spontané, sinon anarchique, pour tenter de le fixer fermement sous le contrôle institutionnel de l'autorité politique et ecclésiastique.

Les cahiers de la Bonne Chanson confondent le folklore avec tout ce qui est nostalgique, moralisateur et réactionnaire - bref, ils présentent le folklore comme élément d'une idéologie qui rejette la réalité moderne et qui n'a aucune signification pour une population qui, même à l'époque, devenait de plus en plus urbaine et complexe. On est en droit de se poser la question à savoir si les cahiers de la Bonne Chanson n'auraient pas - contrairement aux attentes de son éditeur - servit à marginaliser davantage les chansons de tradition orale que d'assurer leur survie.

L'œuvre de l'abbé Gadbois aurait eu un autre effet réducteur sur le répertoire de chansons folkloriques circulant dans la population générale. Rappelons qu'un chansonnier n'est pas une étude scientifique ou

22. De Surmont, La Bonne Chanson..., op. cit., p 99.

23. D'après les données des Archives de folklore et ethnologie de l'Université de Sudbury. 
un recueil ethnographique : un chansonnier est un instrument pour faire chanter les gens et, en fait, leur faire chanter un répertoire particulier, un répertoire qu'on juge plus propice à la collectivité que les choix facilement accessibles dans la culture populaire. En plus - pourvu que les chansons passent cet examen idéologique-, on choisit aussi les chansons d'un chansonnier pour leur "chantabilité » et non pour leur rareté ou leur intérêt textuel et musical, puisque le fait de chanter est un acte moral et politique en soi qui renforce les familles, instruit la jeunesse, inspire la loyauté aux valeurs traditionnelles. Or, trois quarts des chansons choisies par Gadbois pour représenter le folklore sont des chansons en laisse et des chansons énumératives - qu'on peut qualifier toutes deux de chansons à répondre - puisqu'elles sont celles que les gens sont les plus aptes à chanter en communauté. Le répertoire traditionnel qui est inclus ne comprend que huit chansons strophiques et trois en forme de dialogue ${ }^{24}$. En mettant l'accent presque exclusivement sur cet aspect du répertoire folklorique, les chansonniers contribuent encore une fois à la perte de diversité, donnant clairement l'impression que la chanson narrative figure à peine dans le répertoire traditionnel.

\section{Les chansonniers de Germain Lemieux}

On ne peut pas comparer la compilation de chansonniers comme ceux de Laverdière, Burque et Gadbois à l'œuvre du folkloriste Germain Lemieux qui, au cours de ses enquêtes de 1948 à 1970, a recueilli plus de 3000 chansons, collecte qui répond à tous les critères d'une recherche ethnologique menée de façon rigoureuse et méticuleuse ${ }^{25}$. Si le père Lemieux avait lui aussi ses idées quant au folklore et à la survivance de la langue et de la culture de la communauté franco-ontarienne, cela ne l'a pas empêché de documenter fidèlement ce qui survivait du répertoire traditionnel apporté en territoire ontarien par les colons québécois de la fin du xixe et du début du $x x^{e}$ siècle. Mais sa collection demeure pour la plupart inédite et inconnue ; le père Lemieux n'a publié qu'une partie minime de son corpus dans quatre petits chansonniers comprenant à peine 150 chansons. Il s'agit de Folklore franco-ontarien I et II (1949 et 1950) et Chansonnier francoontarien l et II (1974 et 1975) ${ }^{26}$. La présentation de ces petits bouquins

24. Voir Marcel Bénéteau, « Aspects de la tradition orale comme marqueurs d'identité culturelle : le vocabulaire et la chanson traditionnelle des francophones $\mathrm{du}$ Détroit ", Québec, thèse de doctorat, Université Laval, 2000.

25. Collection déposée au Centre franco-ontarien de folklore à Sudbury, Ontario. Le corpus de chansons a récemment été catalogué d'après les données du Catalogue Laforte par Marcel Bénéteau et Lucie Beaupré.

26. Germain Lemieux, Folklore franco-ontarien. Chansons, Sudbury, La Société historique du Nouvel-Ontario (SHNo), "Documents historiques » n 17, 1949, 48 p. ; Folklore franco-ontarien. Chansons II, Sudbury, Sнno, 1950, 48 p. ; 
démontre cependant que le but de ces chansonniers n'est pas tellement différent de celui de leurs prédécesseurs.

Il est évident, tout d'abord, que le père Lemieux a fait lui aussi un " choix de chansons " pour ses chansonniers. L'analyse de sa collection révèle qu'il a recueilli deux fois plus de chansons types dans la catégorie strophique que dans les chansons en laisse. Mais il donne quand même la place d'honneur aux " chansons à répondre » (deux tiers du répertoire publié). Lui aussi semble miser surtout sur la « chantabilité » des chansons - après tout, c'est le but d'un chansonnier. Mais c'est dans ses propres paroles qu'on note une forte sympathie avec les préoccupations des autres compilateurs de chansonniers. Dès son premier petit cahier de Folklore franco-ontarien, le père Lemieux explique son but en publiant un chansonnier :

[...] nous avons remarqué que tous ceux auprès desquels nous avons sollicité ces chansons d'autrefois déplorent que la plupart des jeunes de leur entourage préfère à leur répertoire ancestral la chanson de jazz. Comme ils sont heureux de nous entendre dire que, dorénavant, une foule de jeunes vont répéter ces chants entraînants pour égayer leurs réunions ou rythmer leur marche ! ${ }^{27}$

Dans le deuxième volume, il affirme à nouveau : «C'est pour favoriser ces fêtes intimes et faire revivre la joie saine et simple des ancêtres que nous cueillons et publions nos chansons. ${ }^{28}$ " Et dans le premier volume du Chansonnier franco-ontarien : "Ce recueil voudrait redonner à notre population cette fierté patriotique dont débordait nos ancêtres dans leurs randonnées, leurs travaux ou leurs délassements de groupe. ${ }^{29}$ »

Il est évident que le père Lemieux ne considère pas la sauvegarde et la diffusion du folklore comme des actions neutres. Il n'a d'ailleurs jamais caché les motivations pédagogiques de son travail30. Mais c'est dans une petite étude sur la chanson traditionnelle publiée en 1964 - Chanteurs

Chansonnier franco-ontarien I, Sudbury, Centre franco-ontarien de folklore et SHNo, "Documents historiques " ${ }^{\circ} 64,1974,135$ p. ; Chansonnier franco-ontarien ॥, Sudbury, Centre franco-ontarien de folklore et SHNO, "Documents historiques » $n^{\circ} 66,1975,140 \mathrm{p}$.

27. Germain Lemieux, Folklore franco-ontarien. Chansons, p. 4.

28. Germain Lemieux, Folklore franco-ontarien. Chansons II, p. 6.

29. Germain Lemieux, Chansonnier franco-ontarien I, p. 1.

30. Voir, par exemple, Germain Lemieux, « Mon projet folklorique cinquante ans plus tard ", dans Jean-Pierre Pichette (dir.), L'Éuvre de Germain Lemieux, s.j. Bilan de l'ethnologie en Ontario français, Sudbury, Prise de parole et Centre francoontarien de folklore, $1993,521 \mathrm{p}$. 
franco-ontariens et leurs chansons-que Germain Lemieux exprime le plus clairement la vocation qu'il attribue au folklore :

Comprenons-nous l'importance pour nous, Canadiens français, de mieux connaître notre folklore et de nous y cramponner comme à un ponton de sauvetage ? C'est une source de fierté, un bain d'énergie patriotique, un rempart contre l'anglicisation et l'américanisation. En chantant nos joies et nos peines dans le monde ancestral, nous réintégrons cette âme française qui, en se canadianisant, devint celle des laborieux défricheurs, des joyeux découvreurs, des malheureux déportés et des lutteurs persévérants. Pourquoi importer des États-Unis des danses négro-américaines pour occuper les loisirs de Canadiens français dont les ancêtres ont propagé le Menuet et le Salut-Mesdames ? Pourquoi acheter à des criailleurs maladifs des chansons qui tentent de faire disparaître le rythme intérieur et l'équilibre des gens normaux ? [...] Nous serons Canadiens français dans la mesure où nous suivrons les traces des générations passées : gaîté française, sens artistique, clarté d'expression, retenue chrétienne et respect des valeurs que nous ont léguées nos pères. ${ }^{31}$

Admettons que le père Lemieux se fie à la sagesse du peuple beaucoup plus que les abbés Burque et Gadbois. Il ne voit aucun besoin d'améliorer ou de censurer le folklore, bien que l'autocensure pratiquée par ses informateurs ait peut-être réduit ce besoin. Mais, quant au rôle du folklore dans la société et l'usage des chansonniers, il affiche décidément un point de vue rétrograde. La méthodologie d'un collecteur formé par Luc Lacourcière est évidemment très différente de celles des simples compilateurs de chansonniers. Mais le modèle établi par ces religieux semble avoir exercé son influence même chez l'un des grands prêtres collecteurs. 


\section{Annexe I-Vive la Canadienne}

Version d'Ernest Gagnon, p. $25-26$

Vive la Canadienne, Vole mon cœur vole, Vive la Canadienne et ses jolis yeux doux.

Et ses jolis yeux doux, doux, doux

Et ses jolis yeux doux.

Nous la menons aux noces Dans tous ses beaux atours.

Là, nous jasons sans gêne, Nous nous amusons tous.

Nous faisons bonne chère, Et nous avons bon goût.

On danse avec nos blondes ; Nous changeons tour à tour.

On passe la carafe, Nous buvons tous un coup.

Mais le bonheur augmente Quand nous sommes tous soûls.

Alors toute la terre Nous appartient en tout!

Nous nous levons de table, Le cœur en amadou.

Nous finissons par mettre Tout sens dessus dessous.

Ainsi le temps se passe, Il est vraiment bien doux.
Version de l'abbé Burque, p. 51-52

Vive la Canadienne, Vole, mon cœur, vole, Et ses jolis yeux doux.

Et ses jolis yeux doux, doux, doux

Et ses jolis yeux doux.

Femme forte et chrétienne, Elle apprend à sa fille Elle nous charme tous.

Elle est fine et gentille, Belle dans tous les goûts.

À ménager les sous.

Elle fait à la merveille

La bonne soupe aux chous. [sic]

Elle rayonne et brille,

Elle est bien sans pareille

Avec ou sans bijous [sic].

Pour soigner rhume et toux.

Nous l'aimons à l'extrême, On adore ses tartes, D’amour tendre et jaloux.

Son beurre et ses ragoûts.

Elle est reine suprême, Des cœurs à ses genoux.

Elle a toujours aux cartes Les mains pleines d'atouts.

C'est à qui la marie, Jusqu'à l'heure dernière, Les garçons en sont fous. Sa vie est toute à nous.

Par sa riche industrie, Elle joint les deux bouts.

Ce n'est qu'au cimetière Que son règne est dissous.

Que d'enfants elle donne À son joyeux époux !

Allons fleurir sa tombe, Un grand cœur est dessous.

La famille foisonne En petits manitous. Que notre pleur y tombe, Plein de souvenez-vous.
Elle fait à l'aiguille Nos habits, nos surtouts. 


\section{Annexe II - Par derrière chez ma tante (Gai lon la, gai le rosier)}

\section{Version Gagnon, p. 51-52}

Par derrièr' chez ma tante

Lui ya-t-un bois joli ;

Le rossignol y chante

Et le jour et la nuit.

Gai lon la, gai le rosier,

Du joli mois de mai.

Le rossignol y chante

Et le jour et la nuit.

Il y chante pour ces belles

Qui n'ont pas de mari.

II ne chante pas pour moi

Car j'en ai-t-un joli.

II n'est point dans la danse, Il est bien loin d'ici.

Il est dans la Hollande :

Les Hollandais l'ont pris.

- Que donneriez-vous, belle, Qui l'amèn'rait ici ?

- Je donnerais Versailles, Paris et Saint-Denis, Et la claire fontaine De mon jardin joli.

\section{Version Burque, p. 86-87}

Au bois qui nous enchante, La peine me conduit; Le rossignol y chante Et le jour et la nuit.

\section{Gai lon la, joli rosier} Du joli mois de mai.

Il chante pour ses dames Qui se meurent d'ennui, Quand, désertant leurs âmes, Le bonheur s'est enfui.

Moi je pleure l'absence De mon brave mari : Il n'est point dans la France, Il est bien loin d'ici.

Trop vaillant à la guerre, Les ennemis l'ont pris : Terminer sa misère Je voudrais à tout prix.

- Que donneriez-vous, belle, Qu'il fût par nous repris, Et que, toujours fidèle, Il fût à vous remis?

- Je donnerais la Seine, Le Pérou, le Chili, Et la claire fontaine De mon jardin joli.

Je donnerais Versailles, Paris et Saint-Denis, La Chine et ses murailles, Tous les biens réunis.

- Venez donc voir, madame, Votre galant mari : L'objet de votre flamme, Regardez, le voici.
- 0 jour plein d'allégresse Et mille fois béni ! Adieu, peine et tristesse ! Mon malheur est fini !

Aussitôt elle embrasse Son bel époux chéri : Le bonheur a pris place Dans son cœur attendri.

Note : Introduction

à la chanson

"Le texte populaire de cette bonne vieille chanson est malheureusement défectueux. Cela est regrettable précisément à cause de la valeur intrinsèque et de l'importance nationale de cette pièce, une des plus belles de notre répertoire. Le texte proposé ici est une exposition beaucoup plus logique du sujet, avec le même caractère de vivacité et de simplicité. Il se chante tout aussi bien, et l'esprit en est plus satisfait. / Qu'on l'essaye, et l'on verra. » 